\title{
Etude du régime alimentaire de cinq espèces de Cladocères dans la retenue Lalla Takerkoust (Maroc)
}

\author{
A. Tifnouti ${ }^{1}$ \\ O. Chérifi2 \\ A. Chifaa ${ }^{2}$
}

Mots clés : régime alimentaire, Cladocères, phytoplancton, herbivore.

L'examen du contenu du tube digestif des Cladocères de la retenue Lalla Takerkoust (Maroc) indique clairement la dominance phytoplanctonophage des espèces. Toutes les espèces sont des filtreuses herbivores, se nourrissant essentiellement d'algues. Cependant, chez les classes de petite taille il y a utilisation, en plus des algues, de détritus et de bactéries. La spécificité alimentaire est plus ou moins marquée selon les espèces, surtout chez les Daphnies. Diaphanosoma brachyurum (Lievin 1848) et Ceriodaphnia dubia (Richard 1894) apparaissent comme des filtreurs passifs.

Certaines espèces telles $C$. dubia, D. brachyurum et Bosmina longirostris (O.F. Muller 1785) ont un éventail alimentaire identique et plus étroit que les autres espèces. Elles se nourrissent de Cyclotella ocellata (Pantocksek), de Schroederia sp. et d'Euglena sp. Par ailleurs, Daphnia lumholtzi (Sars 1886) et Daphnia pulex (Leydig 1860), espèces de grande taille, présentent un spectre alimentaire plus large. En effet, en plus de Cyclotella, Schroederia et Euglena sp, D. lumboltzi s'alimente de Cosmarium laeve (Rabenhorst) alors que $D$. pulex se nourrit de Cyanophycées et de Dinophycées.

La taille des animaux semble être un élément important dans la détermination de la taille des particules ingérées, ce qui confirme les résultats de Burns (1968). Les stades jeunes consomment principalement des micro-algues.

\section{A study of the diet of five species of Cladocera in the reservoir Lalla Takerkoust (Morocco)}

Keywords : diet, Cladocera, phytoplankton, herbivore.

The examination of the gut contents of cladocerans from the reservoir Lalla Takerkoust (Morocco) showed clearly the dominance of phytoplankton feeding by these species. All species are herbivorous filter-feeders that feed on algae. However, in the smallest size classes, detritus and bacteria are utilized as well as algae. The specific nature of the diet is more or less marked amongst the species, especially daphniids. Diaphanosoma brachyurum (Lievin 1848) and Ceriodaphnia dubia (Richard 1894) appear to be passive filterers. Certain species, such as C. dubia, D. brachyurum and Bosmina longirostris (O.F. Müller 1785) have an alimentary fan that is identical and more closed than that of other species. They feed on Cyclotella ocellata (Pantocksek), Schroederia sp. and Euglena sp. In addition, Daphnia lumholtzi (Sars 1886) and Daphnia pulex (Leydig 1860), both species of large size, have a larger range of diet. As well as Cyclotella, Schroederia and Euglena sp., D. lumholtzi feeds on Cosmarium laeve (Rabenhorst) whereas D. pulex feeds on Cyaophyceae and Dinophycae. Animal size seems to be an important element in determining the size of ingested particles, which confirms the results of Burns (1968). The young stages consume chiefly micro-algae.

\section{Introduction}

L'objectif de ce travail est d'analyser (après avoir étudié l'évolution saisonnière des ressources alimentaires c'est-à-dire le phytoplancton, Chérifi 1992),

\footnotetext{
1. Laboratoire d'Hydrobiologie, Département de Biologie, Faculté des Sciences Semlalia. B.P. S 15, Marrakech, Maroc.

2. Laboratoire d'Algologie, Département de Biologie, Faculté des Sciences Semlalia. B.P. S 15, Marrakech, Maroc.
}

le régime alimentaire des Cladocères qui constituent le peuplement zooplanctonique le plus important dans la retenue Lalla Takerkoust et de préciser la place qu'occupent ces animaux dans le réseau trophique du lac et améliorer nos connaissances sur l'écologie nutritionnelle des espèces de Cladocères.

Le statut trophique des lacs réservoirs est en grande partie déterminé par le renouvellement de l'eau, les apports du bassin versant, la composition du peuplement pisciaire et surtout par les rapports 
producteurs-consommateurs. Le zooplancton, en particulier les Cladocères, joue un rôle essentiel dans la régulation des populations algales en consommant des quantités importantes d'algues, provoquant ainsi une diminution de la biomasse. Ce phénomène se traduit par une forte augmentation de la transparence aboutissant à une phase des eaux claires, couramment observée dans les lacs tempérés par de nombreux auteurs (Edmondson 1957, Lampert 1974 et 1978, Sommer 1985, Sommer et al. 1986).

La température, dont les effets ont été examinés antérieurement (Tifnouti et al. 1993) et la nourriture constituent les facteurs essentiels qui régulent la croissance des populations de Cladocères.

\section{Caractères généraux du réservoir}

Le lac de Lalla Takerkoust est situé à environ trente-cinq kilomètres au sud-sud-ouest de Marrakech. Il a été construit entre 1929 et 1935 pour l'irrigation et la production d'énergie électrique. Le réservoir, de $7 \mathrm{~km}$ de long et $1 \mathrm{~km}$ de largeur maximum, a une capacité d'environ $62.10^{6} \mathrm{~m}^{3}$.

Situé à $600 \mathrm{~m}$ d'altitude, il est alimenté par les eaux de l'oued N'fis (affluent de l'oued Tensift) qui prend sa source au plateau de Tichka (3 $351 \mathrm{~m}$ ) dans le Haut Atlas. La superficie drainée est de $1700 \mathrm{~km}^{2}$ et l'essentiel des précipitations se concentre durant la saison froide. Les eaux du réservoir sont moyennement minéralisées ( $<640 \mu \mathrm{S} / \mathrm{cm}$ ) (Chérifi 1990).

Les vents dominants sont de deux types : 1 - en été, le chergui, sec et chaud, soufflant du sud-est et le siroco venant du sud, 2 - en hiver, le gharbi, froid et humide, de secteur sud-ouest à nord-ouest.

Le développement des macrophytes est réduit à une frange littorale dans la zone amont de profondeur limitée $(0,70$ à $3 \mathrm{~m})$. La faune pisciaire est essentiellement représentée par des carpes communes (environ $60 \%$ du stock) accompagnées de barbeaux, gardons, anguilles, carpes herbivores (carpes argentées).

\section{Méthodes d'étude}

\subsection{Régime alimentaire}

Plusieurs méthodes peuvent être utilisées pour analyser les contenus stomacaux (Hynes 1950). Parmi ces méthodes, nous avons utilisé celle qui consiste à dénombrer les estomacs dans lesquels une

catégorie de proies est présente, puis à exprimer ce nombre en pourcentage par rapport au nombre total d'estomacs contenant de la nourriture. Le choix de cette méthode parmi les autres est surtout un problème technique vu qu'au Maroc, en général, nous ne pouvons utiliser de radiotraceurs.

L'étude a été effectuée lors des périodes d'abondance des espèces dans la retenue. En effet, à part $D$. pulex et $B$. longirostris dont les évolutions quantitatives saisonnières se situent en hiver, les autres espèces présentent un maximum d'abondance au printemps.

\subsection{Classes de taille et biomasses}

Afin d'étudier le comportement des espèces visà-vis de la nourriture en fonction de l'âge, l'étude a été effectuée pour chaque classe de taille des cinq espèces. La différenciation des classes de taille est obtenue pour chaque espèce de Cladocère récoltée dans le lac. Ainsi, dans un même prélèvement, la taille de 200 individus d'une même espèce est mesurée sous loupe binoculaire avec un micromètre gradué. L'opération est répétée trois fois à des dates différentes. Les courbes taille-abondance nous ont permis de choisir, pour chaque espèce, un certain nombre de classes d'âge.

Ainsi pour Daphnia lumholtzi (Sars 1886) et Daphnia pulex (Leydig 1860), 4 classes d'âge ont été définies :

$\begin{array}{cc}\text { D. lumholtzi } & \text { D. pulex } \\ \mathrm{C} 1:[0,50-0,92 \mathrm{~mm}] & \mathrm{C} 1:[0,40-0,80 \mathrm{~mm}] \\ \mathrm{C} 2:[0,92-1,28 \mathrm{~mm}] & \mathrm{C} 2:[0,80-1,20 \mathrm{~mm}] \\ \mathrm{C} 3:[1,28-1,66 \mathrm{~mm}] & \mathrm{C} 3:[1,20-1,60 \mathrm{~mm}] \\ & \text { préadultes } \\ \mathrm{C} 4:[1,66-2.04, \mathrm{~mm}] & \mathrm{C} 4:[1,60-2,02 \mathrm{~mm}] \\ & \text { adultes et femelles } \\ & \text { ovigères }\end{array}$

3 classes pour Ceriodaphnia dubia (Richard 1894) et Diaphanosoma brachyurum (Lievin 1848).

\section{C. dubia}

$\mathrm{C} 1:[0,30-0,54 \mathrm{~mm}]$

$\mathrm{C} 2:[0,54-0,84 \mathrm{~mm}]$

C3 : $[0,84-1,13 \mathrm{~mm}]$

\section{D. brachyurum}

$$
\begin{aligned}
\mathrm{C} 1: & {[0,36-0,67 \mathrm{~mm}] } \\
\mathrm{C} 2: & {[0,67-0,97 \mathrm{~mm}] } \\
& \text { préadultes } \\
\mathrm{C} 3: & {[0,97-1,30 \mathrm{~mm}] } \\
& \begin{array}{l}
\text { adultes et femelles } \\
\text { ovigères }
\end{array}
\end{aligned}
$$

et deux classes pour $B$. longirostris,

$\mathrm{C} 1:[0,20-0,42 \mathrm{~mm}]$

$\mathrm{C} 2:[0,42-0,63 \mathrm{~mm}]$ adultes et femelles ovigères 
Les mesures de la taille du corps ont été effectuées sans tenir compte des épines postérieures ou antérieures.

Pour les biomasses, des pesées ont été effectuées pour chaque classe de taille des cinq espèces de Cladocères ; les poids frais et sec moyen d'un individu sont calculés pour chaque classe de taille précédemment définie.

Les biomasses des espèces ont été calculées en effectuant des pesées pour chaque espèce voire pour chaque classe de taille d'une même espèce et en établissant des équations de régression taille-poids sec des Cladocères et qui sont propres à la retenue Lalla Takerkoust.

Les poids frais et sec d'un individu sont calculés selon les méthodes décrites par Pikryl (1980) et Pont (1983).

\subsection{Indices de sélection}

Plusieurs indices ont été proposés afin d'exprimer la sélection des proies :

- L'indice d'Ivlev (1961), selon plusieurs auteurs (Hutchinson 1971, Obrien \& Vinyard 1978, Guissani 1974), présente l'inconvénient d'être significativement biaisé lorsque les effectifs de la proie dans l'estomac du prédateur et dans l'habitat sont très inégaux (Jacobs 1974, Chesson 1978).

- L'indice proposé par Strauss (1979) (Linear food selection index) présente l'inconvénient de ne pas permettre de comparer les sélectivités pour une proie particulière entre les échantillons où son abondance est très variable, soit dans la nourriture ingérée, soit dans le milieu (Lazarro 1987).

- L'indice de Paloheimo (1979) qui selon Lechowicz (1982), a le défaut de ne pas être symétrique par rapport à la valeur zéro représentant l'absence de sélection.

Pourriot et al. (1982) ont utilisé ces deux derniers indices pour la mesure des préférences alimentaires du Rotifère Asplanchna girodi.

Le faible nombre de proies trouvées dans les estomacs des espèces exclut l'utilisation des méthodes factorielles d'analyse.

Parmi ces indices, nous avons retenu l'indice d'Ivlev, le plus fréquemment utilisé et qui est défini par l'équation suivante :

$$
\mathrm{Ei}=\frac{\mathrm{ri}-\mathrm{pi}}{\mathrm{ri}+\mathrm{pi}}
$$

où ri est le pourcentage d'une algue consommée $\mathrm{i}$ (nannoplancton) dans le contenu stomacal d'une espèce de Cladocères (nombre d'individus de i par rapport au nombre total d'individus) et pi, son pourcentage dans le milieu naturel, calculé en ne considérant que les algues possibles, c'est-à-dire présentes dans le contenu stomacal.

L'indice Ei varie de -1 à +1 . Quand Ei. est voisin de +1 , on considère que l'algue est activement recherchée dans le milieu. Si l'indice est négatif (sélection négative) on considère que c'est une contre sélection. Par ailleurs, si l'indice est égal à 0 , c'est une non sélection.

\section{Résultats}

Les résultats obtenus sont exprimés en pourcentage d'espèces algales rencontrées dans le tube digestif des espèces de Cladocères. Pour les différentes classes de taille, les résultats sont exprimés en nombre de cellules algales par microgramme de poids sec de chaque stade.

\subsection{Daphnia Iumholtzi}

Au cours de 1991, l'espèce était abondante de mai à juillet 1991 avec un pic au mois de mai. L'étude du contenu du tube digestif de ce Cladocère a été effectuée à deux dates différentes. Dans la retenue, le peuplement algal était dominé par la Diatomée Cyclotella ocellata qui constitue plus de $94 \%$ du phytoplancton total. En revanche, l'Euchlorophycée Schroederia ne représente que $5 \%$ (Fig. 1).

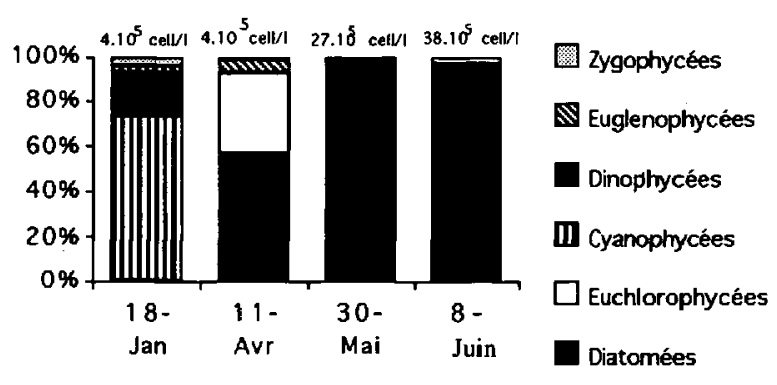

Fig. 1. Densité du phytoplancton total de la retenue Lalla Takerkoust ( 10 cellules $/ 1$ ) correspondant aux périodes d'étude du zooplancton.

Fig. 1. Density of total phytoplancton in L. T. lake ( 10 cells $/ 1)$ during the period of the zooplankton study. 
Les résultats obtenus montrent que pour les deux dates étudiées, le régime alimentaire de $D$. lumholtzi $i$ est très différent. En effet, au cours de la première période, la part majeure d'algues ingérée est constituée par les petites Cyclotella ocellata qui semblent être une bonne nourriture pour $D$. lumholtzi. L'examen des pourcentages indique $61 \%$ de Cyclotelles dans le tube digestif des Daphnies alors que Schroederia ne représente que $30 \%$ (Fig. 2 a). Parmi les algues à faible pourcentage, on peut remarquer les Zygophycées (Cosmarium subprotumidum) et les Euglénophycées (Euglena sp.) sont rencontrées dans le tube digestif avec des pourcentages très faibles. Il faut cependant noter que cette dernière fraction est très peu abondante dans la retenue en cette période.

Lors de la deuxième période, l'Euchlorophycée Schroederia qui représente $94 \%$ de la nourriture algale déterminée dans le tube digestif de ce Cladocère, constitue la nourriture de base. En revanche, les Cyclotelles ne représentent que 5,6 \% (Fig. 2a), malgré leur pourcentage important dans le lac (plus de $90 \%$ ).

L'étude du contenu du tube digestif des différents stades montre que les petites tailles (CL 1) consomment, en général, plus que les femelles pré-adultes et adultes, surtout au cours de la deuxième période (Fig. 2b). De même, le régime des adultes est différent de celui des jeunes. En effet les Zygophycées ne semblent pas être une source de nourriture pour les petites tailles (CL1, CL2 et CL3), contrairement aux Euglénophycées présentes dans le tube digestif des jeunes stades. En revanche, aucune Euglénophycée n'a été observée dans le tube digestif des femelles adultes alors que les Zygophycées constituent $5,36 \%$ du contenu stomacal (Fig. 2b). Lors de la seconde étude, les jeunes daphnies (CL1 et CL2) ne se nourrissent pas de Cyclotelles, mais uniquement de Schroedoria (Fig. 2b).

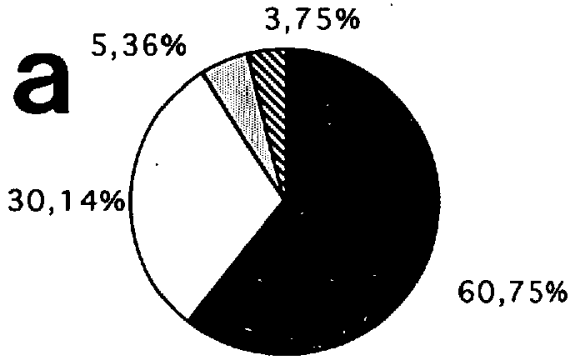

$30 / 5 / 91$

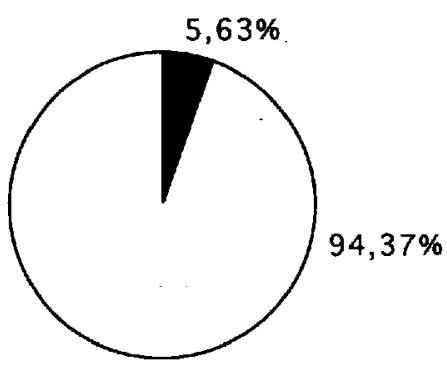

$8 / 6 / 91$
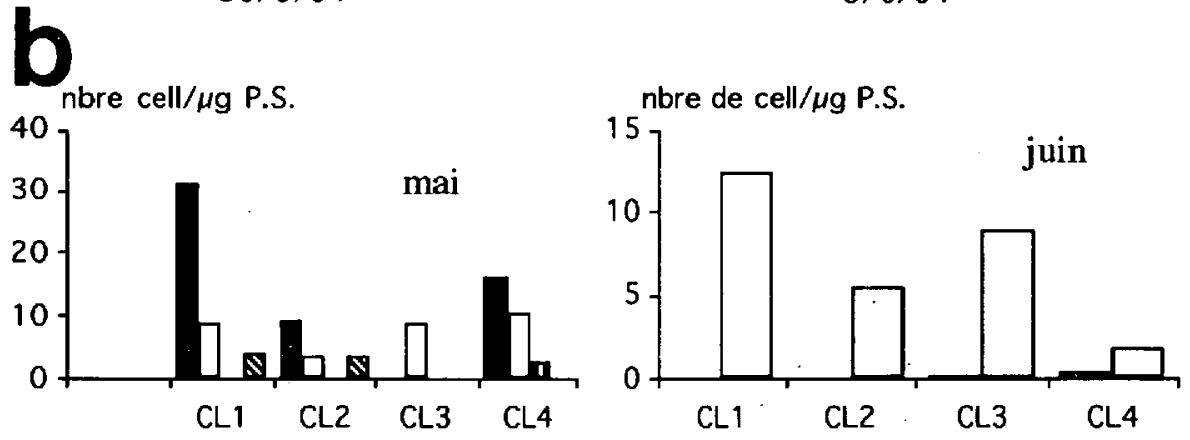

Fig. 2. a. Contenu stomacal moyen de $D$. lumholtzi en pourcentage. Même symboles que Fig. 1 .

b. Contenu stomacal des classes de taille de $D$. lumholtzi en nombre de cellules $/ \mu \mathrm{g}$ de poids sec. Mêmes symboles que Fig. 1.

Fig. 2. a. Mean stomach content of $D$. lumholtzi $(\%)$.

b. Stomach content of different size classes of $D$. lumholtzi expressed as cell numbert/ $\mu \mathrm{g}$ of dry weight. 


\subsection{Daphnia pulex}

Elle est rencontrée dans la retenue tout l'hiver 1991 jusqu'au début du printemps avec les densités importantes en avril.

Le régime alimentaire de l'espèce a été étudié le 11 avril et le 30 mai. En avril, le phytoplancton était dominé par les Euchlorophycées et les Diatomées qui représentent respectivement $43 \%$ et $53 \%$ du peuplement algal du réservoir. En mai, il est dominé par les Diatomées. Les Euchlorophycées sont représentées essentiellement par Schroederia sp., les Diatomées par Cyclotella ocellata (Fig. 1).

L'examen des contenus stomacaux montre que lors de la première étude, les Euchlorophycées constituent la nourriture essentielle de $D$. pulex avec $74 \%$ du contenu stomacal. En second lieu, viennent des Diatomées alors que les Cyanophycées sont présentes dans le tube digestif à de faibles pourcentages, respectivement 17 et $9 \%$ du contenu stomacal (Fig. 3a).
Lors de la deuxième date, les daphnies s'alimentent exclusivement de Diatomées qui constituent $98 \%$ de nourriture observée dans le tube digestif (Fig. 3a).

En regard des classes de taille, on observe que les petites tailles (CL1 et CL2) consomment plus que les pré-adultes (CL3) et adultes (CL4) sauf au cours de la deuxième période où les adultes consomment beaucoup plus que les autres stades. On observe aussi, lors de la première étude, une différence de nourriture entre les différentes classes de l'espèce (Fig. 3b) : seules les daphnies de grande taille s'alimentent de Dinophycées. De même, aucune Cyanophycée n'a été déterminée chez les premiers stades (CL1). Au cours de la deuxième étude, le nombre d'algues broutées est proportionnel à la classe de taille (Fig. 3b). On observe également une prédominance des Euchlorophycées qui peut s'expliquer par leur abondance dans le milieu.
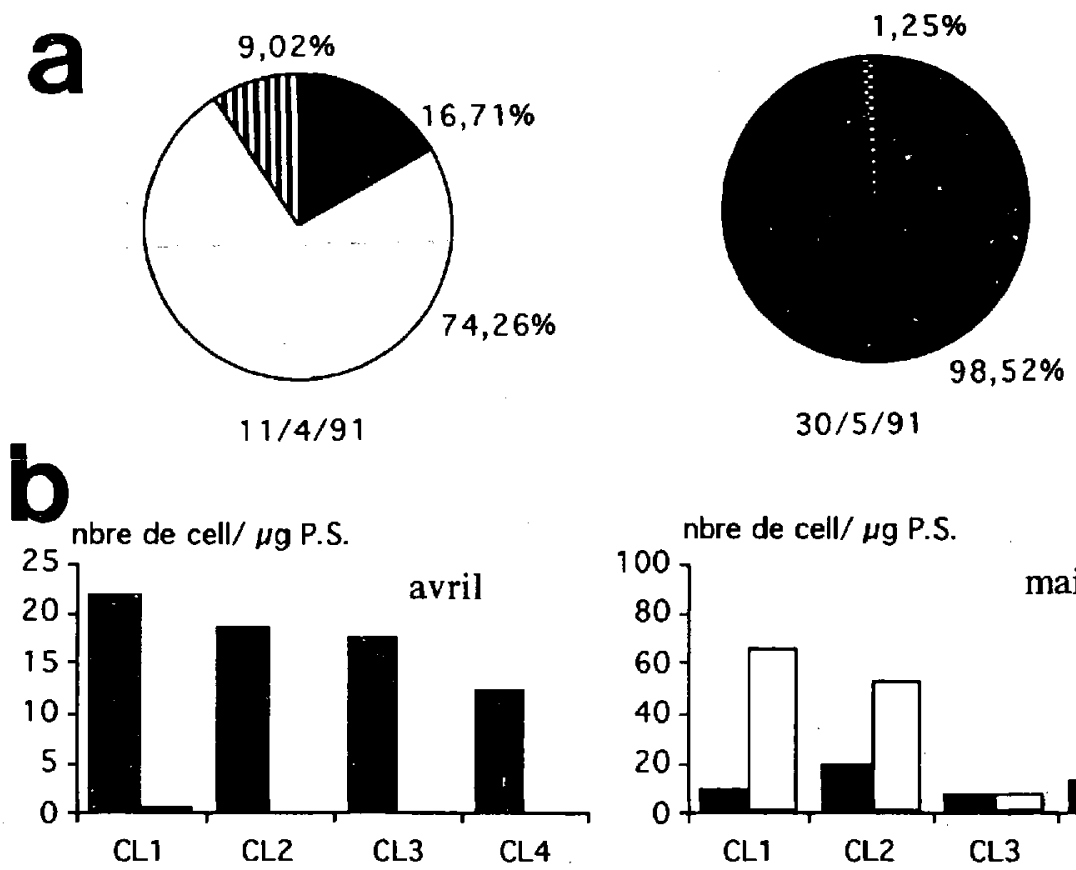

$30 / 5 / 91$
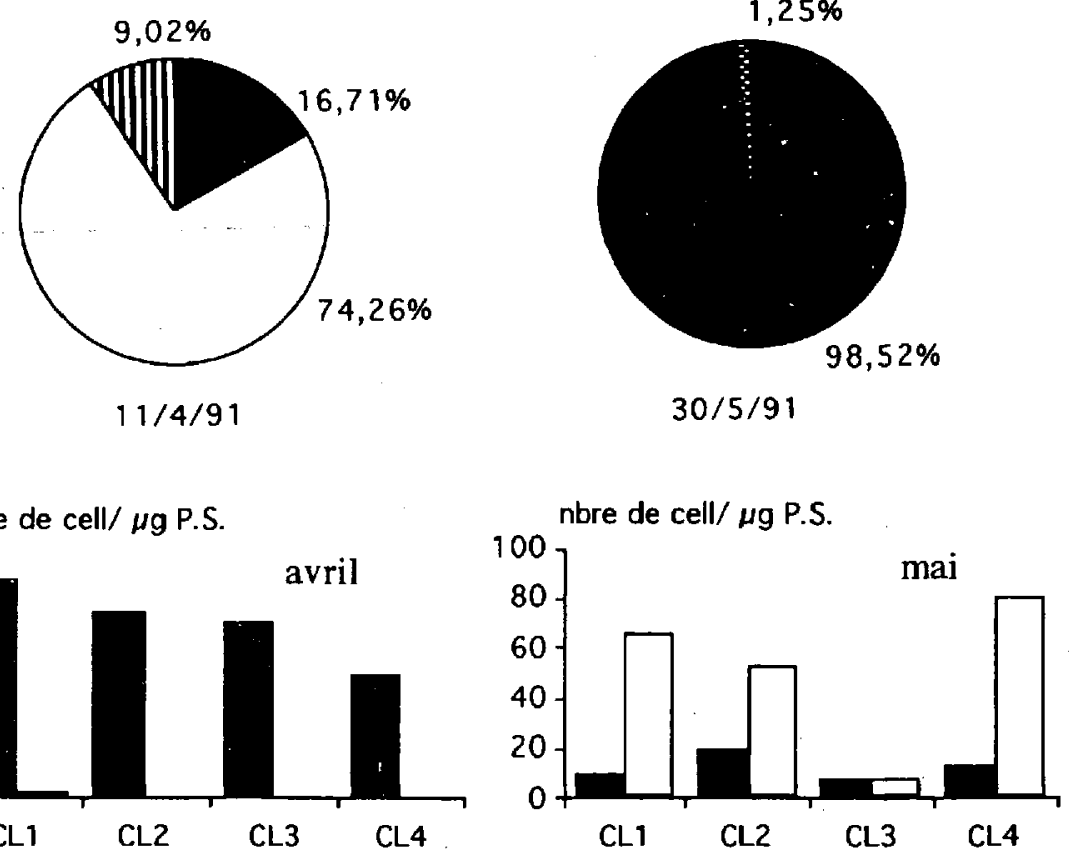

Fig. 3. a. Contenu stomacal moyen de $D$. pulex en pourcentage. Même symboles que Fig. 1 .

b. Contenu stomacal des classes de taille de $D$. pulex en nombre de cellules/ $\mu \mathrm{g}$ de poids sec. Mêmes symboles que Fig. 1 .

Fig. 3. a. Mean stomach content of $D$. pulex $(\%)$.

b. Stomach content of different size classes of $D$. pulex expressed as cell number $/ \mu \mathrm{g}$ of dry weight. 


\subsection{Ceriodaphnia dubia}

Lors de l'examen du contenu stomacal de l'espèce, abondante dans la retenue, le phytoplancton était dominé en avril par un mélange de Diatomées et d'Euchlorophycées (respectivement $58 \%$ et $36 \%$ ). $\mathrm{Au}$ mois de mai, on observe une dominance des Diatomées $(83 \%)$ alors que les Euchlorophycées ne représentent que $4 \%$. Les Diatomées sont exclusivement représentées par Cyclotella ocellata (Fig. 1).

$\mathrm{Au}$ cours de la première étude, on observe que l'Euchlorophycée Schroedoria constitue la part majeure de la nourriture (79 \% des algues consommées, Fig. 4a). Les Diatomées sont également assez bien représentées avec $19 \%$ du peuplement global. Parmi les algues accessoires, nous avons observé une faible proportion de l'Euglénophycée Euglena sp., espèce de petite taille et qui constitue $2 \%$ du contenu stomacal (Fig. 4a).
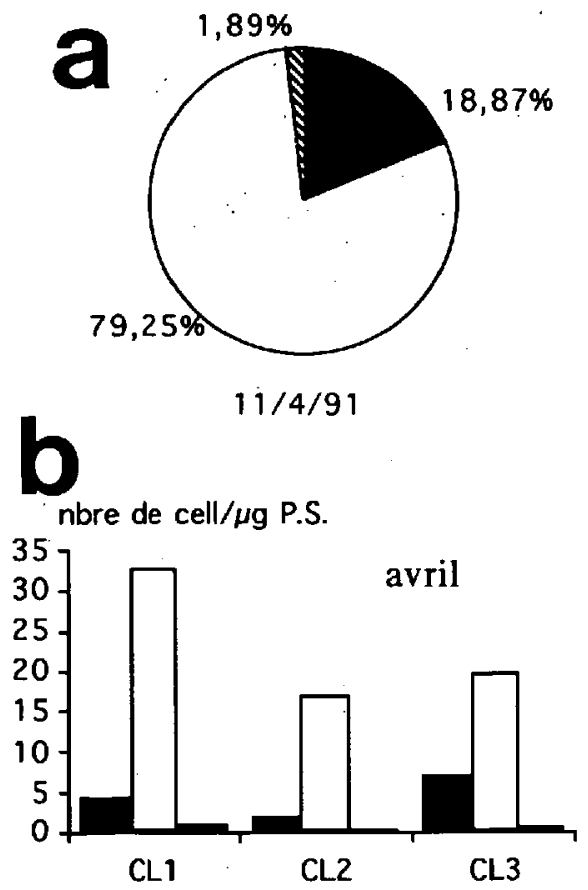

Dans la deuxième période, les Diatomées constituent la nourriture de base de $C$. dubia. La consommation de cette espèce peut atteindre jusqu'à $96 \%$ de Diatomées. Cependant, il faut noter le faible pourcentage des Euchlorophycées dont la fraction est très faible dans le milieu au mois de juin (Fig. 1).

Pour les différentes classes de tailles de l'espèce, on constate que le nombre d'algues broutées est inversement proportionnel à la taille. Les jeunes consomment beaucoup plus d'algues que les femelles adultes. Au cours de la deuxième période, les Euchlorophycées ont été rencontrées uniquement dans le tube digestif des femelles adultes (Fig. 4b).

\subsection{Diaphanosoma brachyurum}

L'espèce est plus abondante en été avec un maximum de développement observé en juillet. L'étude du régime alimentaire de ce Cladocère a été faite aux mois de mai et juin 1991 où domine la Diatomée Cyclotella ocellata (Fig. 1).
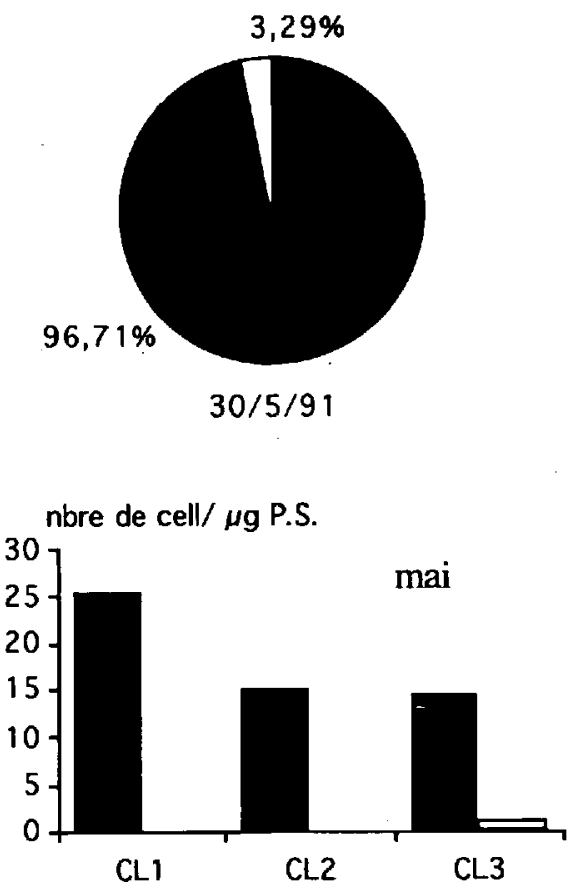

Fig. 4. a. Contenu stomacal moyen de $C$. dubia en pourcentage. Mêmes symboles que Fig. 1.

b. Contenu stomacal des classes de taille de $C$. dubia en nombre de cellules/ $\mu \mathrm{g}$ de poids sec. Mêmes symboles que Fig. 1.

Fig. 4. a. Mean stomach content of $D$. dubia (\%).

b. Stomach content of different size classes of $C$. dubia expressed as cell number $/ \mu \mathrm{g}$ of dry weight. 
Au cours du mois de mai, on observe une consommation plus marquée des Cyclotelles qui peut atteindre jusqu'à $96 \%$ du phytoplancton algal global (Fig. 5a).

En mai, la quantité d'algues consommées est à peu près identique pour toutes les classes de taille et proche de 10 cellules par $\mu \mathrm{g}$ de poids sec.

Au cours du mois de juin, les Euchlorophycées consommées sont plus importantes chez les femelles adultes (CL3) que chez les autres stades. A l'inverse, on observe une diminution de la consommation des Euglénophycées et des Diatomées du stade CL1 au stade CL3 (Fig. 5b).

\subsection{Bosmina longirostris}

L'examen du tube digestif de cette espèce hivernale a été effectué lors de son maximum d'abondance dans la retenue.
Au cours du mois de janvier, le phytoplancton est caractérisé par du microplancton : Ceratium hirundinella et Peridinium cinctum, qui représentent $27 \%$ du peuplement algal global ; les Euchlorophycées, Oocystis lacustris et Schroederia sp. constituent $13 \%$ du peuplement algal qui comprend en faible pourcentage, la Diatomée Cyclotella ocellata $(7 \%)$; la Zygophycée Cosmarium subprotumidum (3\%) et l'Euglénophycée Euglena sp. (1,5\%; Fig. 1).

Le contenu stomacal de $B$. longirostris indique l'ingestion d'Euchlorophycées ( $57 \%$ ), de Diatomées $(25 \%)$ et d'Euglénophycées (17\%, Fig. 6a).

Pour les classes de tailles, il ressort que les jeunes bosmines se contentent dans leur nourriture de Diatomées et d'Euchlorophycées et ne consomment pas des Cyanophycées qui n'apparaissent que dans le tube digestif des femelles adultes (Fig. 6b).

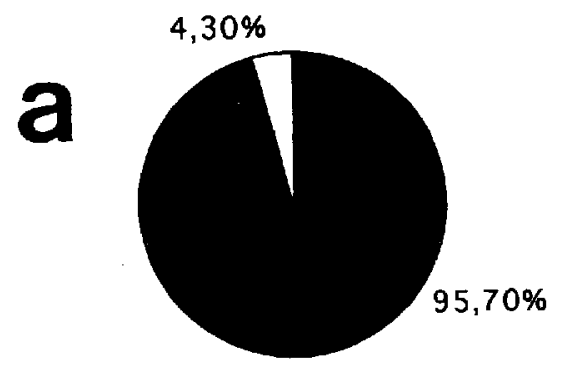

. $30 / 5 / 91$

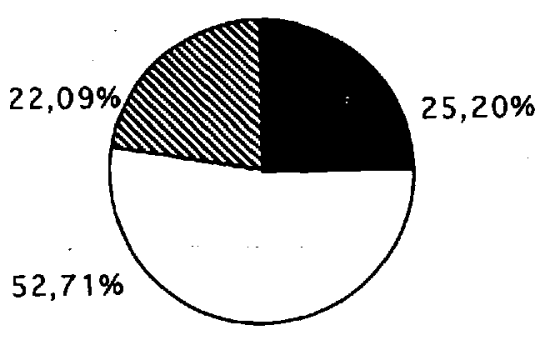

$8 / 6 / 91$
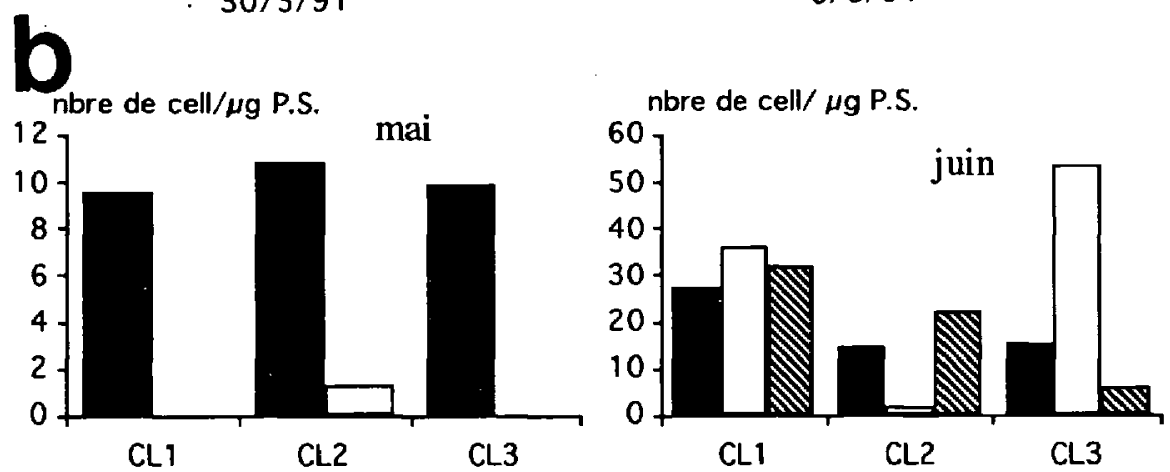

Fig. 5. a. Contenu stomacal moyen de $D$. brachyurum en pourcentage. Mêmes symboles que Fig. 1 .

Fig. 5. a. Contenu stomacal moyen des classes de taille de $D$. brachyurum en nombre de cellules $/ \mu \mathrm{g}$ de poids sec. Mêmes symboles que Fig. 1 .

Fig. 5. b. Mean stomach content of $D$. brachyurum ( $\%$ ).

b. Stomach content of different size classes of $D$. brachyurum expressed as cell number $/ \mu \mathrm{g}$ of dry weight. 


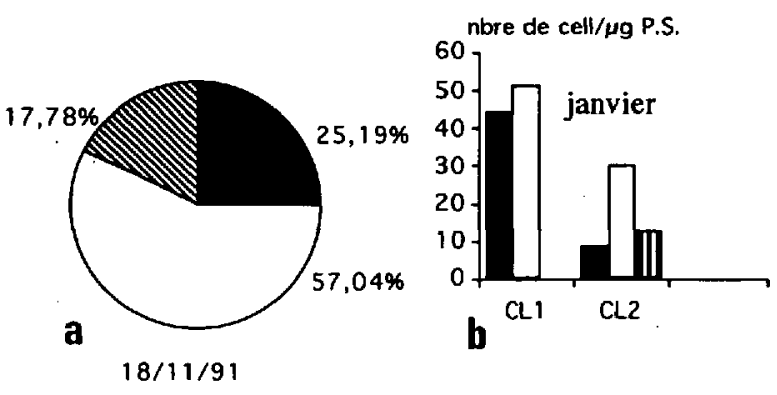

Fig. 6. a. Contenu stomacal moyen de B. longirostris en pourcentage.

b. Contenu stomacal moyen des classes de taille de $B$. longirostris en nombre de cellules/ $\mu \mathrm{g}$ de poids sec. Mêmes symboles que Fig. 1 .

Fig. 6. a. Mean stomach content of $B$. longirostris (\%).

b. Stomach content of different size classes of $B$. longirostris expressed as cell number/ $\mu \mathrm{g}$ of dry weight.

\subsection{Analyse des choix alimentaires}

Les conclusions que l'on peut tirer sur les résultats obtenus par l'indice d'Ivlev sur les algues consommées par les Cladocères montrent que les changements de valeur et/ou de signes des indices rendent malaisée toute interprétation des variations d'indice, en particulier pour les Daphnies (Tableau I).

Il faut souligner les valeurs obtenues pour les cinq espèces de Cladocères et qui sont souvent négatives pour Cyclotella ocellata, alors que sa taille et sa densité dans le plancton, en ces périodes d'étude du régime alimentaire, en feraient d'excellentes ressources alimentaires pour les Cladocères et surtout pour les daphnies reconnues comme étant des filtreurs non sélectifs, ingérant toutes les tailles convenables (Reynolds 1984, Gawler et al. 1986). En même temps, la taille $0-30 \mu \mathrm{m}$ est considérée comme optimale pour les daphnies.

On peut donc en conclure qu'il y a généralement, une contre sélection pour les Cyclotelles, lorsqu'elles ne sont pas les seules composantes du phytoplancton dans le milieu. En revanche, lorsque l'espèce est seule présente dans le milieu (mai 1991), on observe qu'elle n'est pas rejetée mais filtrée indifféremment. Les cas de $D$. brachyurum et $C$. dubia apparaissent plus cohérents (sélectivités proches de 0 ou inférieures à 0,1 ) dans les deux études. Les indices concernant ces deux espèces indiquent l'absence de tout choix, donc une filtration passive de toutes les petites espèces algales.
Pour B. longirostris, on constate que ce Cladocère présente pour Oocystis et Cyclotella des seuils inférieurs au seuil d'indifférence ; il y a donc une contre sélection. En revanche, pour Euglena elle présente un indice proche de +1 , ce qui montre que l'analyse est recherchée activement dans le milieu naturel. Ces résultats confirment ceux de De Mott (1982), qui considère que $B$. longirostris est une espèce sélective qui préfère les algues flagellées (genre Chlamydomonas, Euglena) aux espèces non flagellées.

\section{Discussion et conclusion}

L'examen des pourcentages du tube digestif indique clairement le caractère phytoplanctonophage des Cladocères vivant dans la retenue Lalla Takerkoust. Toutes les espèces sont exclusivement des filtreurs herbivores, se nourrissant d'algues, de détritus et de bactéries sauf $B$. longirostris, considérée comme une espèce sélective (De Mott 1982). Par leurs capacités de filtration élevées, ils participent au recyclage rapide des éléments nutritifs, particulièrement les phosphates. Servant à leur tour de nourriture aux poissons planctonophages, les Caldocères jouent un rôle déterminant et offrent un intérêt primordial dans l'évolution des réservoirs par la manipulation de la chaîne trophique.

Lors de la détermination des algues dans le tube digestif des espèces, plusieurs fragments de détritus (fractions minérales et organiques) ont été observés. Ces observations confirment celles de plusieurs auteurs : outre les micro-algues, les bactéries et les détritus constituent une source alimentaire importante pour les Cladocères.

Heson et al. (1989) notent que les bactéries et les détritus sont les sources de carbone les plus importantes pour la survie des Cladocères. Lampert (1974) montre que les petites tailles $(<1,5 \mathrm{~mm})$ de $D$. pulex mises en présence d'un mélange de bactéries et d'algues, filtrent préférentiellement les bactéries alors que les femelles adultes $(2,5-2,8 \mathrm{~mm})$ consomment algues et bactéries sans discrimination.

Selon Reynolds (1984) et Gawler et al. (1986), les Cladocères herbivores sont reconnus comme des brouteurs non sélectifs, ingérant, semble-t-il sans discrimination, toutes les particules de taille convenable. Brook \& Dodson (1965), Gawler et al. (1986), considèrent la taille $0-30 \mu \mathrm{m}$ des algues; comme taille optimale pour les Daphnies et les Bosmines. 
Tableau 1. Variations de l'indice d'Ivlev des Cladocères de la retenue Lalla Takerkoust.

Table 1. Variations of Ivlev's index for Cladocera of Lalla Takerkoust lake.

D. I : Daphnia lumholtzi - D. p : Daphnia pulex - C. d : Ceriodaphnia dubia - D. b : Diaphanosoma brachyurum - B. I : Bosmina longirostris - S. sp : Spaerocystis $\mathrm{sp}-\mathrm{O} .1$ : Oacystis lacustris - P. d : Pediastrum duplex - S.e : Scenedesmus ecornis - C. $\mathrm{sp}:$ Chlorella $\mathrm{sp}$. - C. o : Cyclotella ocellata C. $\mathrm{s}$ : Cosmarium ubprotumidum - E. sp : Euglena sp. - O. $\mathrm{sp} \mathrm{:} \mathrm{Oscillatora} \mathrm{sp.}$
S. sp
o. 1
P. d
S. e
C. sp
C. 0
C. $\mathbf{s}$
E. sp
o. sp

D. 1

30 mai

8 juin

D. $\mathbf{p}$

11 avril

30 mai

C. d

11 avril

30 mai

D. b

30 mai

8 juin

B. 1

18 janvier

1

10.66

1

1

1

$-0,46$

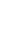

政

$\begin{array}{lllll}0,1 & 1 & -0,88 & & \\ & 1 & -0,22 & 0,84 & 1\end{array}$

1

$-0,40$

0,03

$-0,35$

0,02

0,22

$1 \quad 0,43$

1

$\begin{array}{cc} & 0,01 \\ 1 & -0,58\end{array}$

1

$-0,33$

$-0,006$

0,75

Hesson et al. (1989), notent que les bactéries et les détritus constituent une source de carbone très importante pour la survie du zooplancton en général.

L'étude du contenu stomacal des Cladocères de la retenue Lalla Takerkoust montre que la spécificité alimentaire est plus ou moins marquée selon les espèces, surtout pour les Daphnies. Par ailleurs, les Diaphanosoma et les Ceriodaphnia apparaissent comme des filtreurs passifs. C. dubia, D. brachyurum et $B$. longirostris ont un éventail alimentaire identique et plus étroit que les autres espèces. Elles se nourrissent de Cyclotella, de Schroederia et d'Euglena sp. $D$. lumboltzi et $D$. pulex, espèces de grandes tailles, présentent un spectre alimentaire plus large. En effet, en plus de Cyclotella, de Schroederia et d'Euglena sp., D. lumholtzi s'alimente de Cosmarium alors que $D$. pulex se nourrit de Cyanophycées et de Dinophycées.

Il ressort aussi de ces résultats que la taille des animaux semble être un élément important pour la détermination de la taille des particules ingérées, ce qui confirme les résultats de Burns (1968). Les stades jeunes apparaissent comme principalement consommateurs de micro-algues de petite taille.
C'est le cas de jeunes de $D$. lumholtzi qui ne s'alimentent nullement de Cosmarium et de ceux de $D$. pulex, qui ne consomment pas de Cyanophycées ni de Dinophycées dont les espèces sont lárgement consommées par les adultes. Spurles \& Holtby (1979) mentionnent que la taille ainsi que le mode d'alimentation des organismes peuvent être des descripteurs apportant plus d'information sur la structure de la communauté que la traditionnelle description taxonomique. De nombreux auteurs ont établi une relation linéaire entre la taille des Cladocères et la taille des proies (Burns et al. 1986). Korinek et al. (1980) ont constaté que, selon le milieu, une même espèce peut s'alimenter de différents types de nourriture.

Selon Lampert (1978) et Gliwicz \& Siedlar (1980), les Daphnies sont gênées par le développement d'algues de grande taille.

Au sein d'une même espèce, la quantité d'algues broutées est inversement proportionnelle à la taille.

Ces différences peuvent être expliquées de deux façons :

1) La taille maximale des particules ingérées dépend de l'ouverture buccale : les jeunes Cladocères, de petite taille, ne peuvent s'alimenter d'algues 
de grande taille. C'est le cas de $C$. dubia dont les jeunes (CL1) ne consomment pas d'Euglénophycées alors que les adultes en consomment. Il en est de même pour les espèces de petite taille. C'est le cas de $B$. longirostris, qui ne s'alimente que de petites algues, alors qu'au mois de janvier, le phytoplancton était composé de microplancton de grande taille. Selon Pourriot \& Champs (1982), la gamme de taille des particules ingérées est considérée comme l'un des principaux critères de différenciation des régimes alimentaires, en particulier pour les espèces filtreuses.

A noter aussi que lorsque la taille des Cladocères augmente nous observons une extension du spectre alimentaire vers des particules plus grosses. Ce qui confirme les résultats de Brooks \& Dodson (1965).

Gliwicz (1977) précise à son tour que le maximum observé est en partie fonction de la distance séparant les valves lors de la filtration. Plusieurs auteurs (Gliwicz 1967-1977, Burns 1969, Morgan et al. 1980, Pourriot \& Champs 1982) indiquent des tailles maximales des particules ingérées par les Cladocères: $40 \mu \mathrm{m}$ pour $D$. pulex $; 20 \mu \mathrm{m}$ pour $B$. longirostris et $C$. rectangula.

Dans la retenue Lalla Takerkoust, les tailles des algues ingérées varient entre : $* 8 \mu \mathrm{m}$ et $71 \mu \mathrm{m}$ pour $D$. pulex et $D$. lumholtzi : ${ }^{*} 8 \mu \mathrm{m}$ et $15 \mu \mathrm{m}$ pour $C$. dubia, $D$. brachyurum et $B$. longirostris.

2) La compétition pour la nourriture : les exigences en nutriments des femelles adultes sont très importantes (investissement dans la reproduction), ce qui augmente la consommation du phytoplancton. Dans le cas d'une éventuelle compétition pour la nourriture, les femelles adultes filtrent une plus grande quantité et des algues de plus grande taille que les jeunes. Dans ce sens, on peut citer l'exemple de $D$. lumholtzi chez laquelle on observe dans le premier prélèvement que les petites Daphnies (CL1 et CL2) s'alimentent bien de la Diatomée Cyclotella ocellata, espèce abondante et dominante dans ce prélèvement. Or, dans le deuxième prélèvement, on observe bien que la Diatomée, moins abondante, n'est nullement consommée par les petites daphnies (CL1 et CL2), alors que les femelles adultes (CL4) et sub-adultes (CL3) en utilisent.

L'ensemble de ces observations nous amène en dernier lieu à regrouper les espèces algales récoltées dans la retenue Lalla Takerkoust, en fonction de leur utilisation par les Cladocères :
- Le premier groupe est formé par la Diatomée Cyclotella ocellata et par Schroederia sp. Ce sont des algues de petite taille qui sont ingérées par toutes les espèces, toutes classes confondues. Leur pourcentage d'assimilation peut varier d'une espèce à l'autre mais elles prédominent dans le tube digestif des cinq espèces étudiées. Dans la retenue, Cyclotella est abondante au printemps et en été et Schroederia au printemps.

- Le deuxième groupe est composé par les Euglénophycées représentées par Euglena sp. espèce également de petite taille mais dont la taille dépasse celle du premier groupe. Elle est présente dans la retenue de la fin de l'hiver au début du printemps. Cette espèce est consommée par $D$. lumholtzi, $C$. dubia et par $\boldsymbol{B}$. longirostris. Son pourcentage d'assimilation est beaucoup moins important que celui du premier groupe. Elle est surtout consommée par les femelles adultes.

- Le troisième groupe se compose d'espèces phytoplanctoniques de grande taille et d'importance secondaire dans l'alimentation des espèces de Cladocères étudiés et limité aux seules Daphnies de grande taille. Il s'agit de :

* Cosmarium subprotumidum, Zygophycée abondante dans la retenue en automne et qui est utilisée comme source de nourriture par les femelles adultes de $D$. lumholtzi et de $D$. pulex.

* Peridinium cinctum et Ceratium hirundinella, dont on ne trouve que quelques débris dans le tube digestif. Ces Dinophycées sont consommées uniquement par les grandes tailles de $D$. pulex. Ces deux espèces algales abondent dans la retenue à la fin de l'automne et en hiver.

Enfin, selon Gliwicz (1969), la composition de la nourriture du zooplancton, liée aux espèces présentes, peut être un bon indicateur du statut trophique d'un lac. En effet, la plupart des espèces de Cladocères indicatrices de milieux eutrophes sont des organismes microfiltreurs, qui se nourrissent de bactéries et de détritus. Par ailleurs, dans les milieux oligotrophes, les espèces les mieux représentées sont des organismes macrofiltreurs qui se nourrissent d'algues (Pejler 1983).

En conclusion, l'étude du régime alimentaire des Cladocères, à partir de l'examen du contenu du tube digestif a permis de préciser la place des différentes espèces dans la structure trophique de la retenue. Cependant, un autre point, peu connu dans la 
retenue Lalla Takerkoust principalement et dans tous les barrages du Maroc, en général, reste à éclaircir, celui de l'évaluation des flux des matières au sein de la chaîne trophique qui occupe une place dominante dans l'écologie (Fretwell 1987).

\section{Remerciements}

Les auteurs remercient $\mathbf{R}$. Pourriot et $\mathbf{C}$. Rougier pour leurs suggestions et pour la relecture du manuscrit.

\section{Travaux cités}

Brooks J.L. \& Dodson S.I. 1965. - Predation, body size, and composition of plankton. Science, $150: 28-35$.

Burns C.W. \& Gilbert J.J. 1986. - Effects of daphnie size and density on interference between Daphnia and Keratella cochlearis. Limnol. and Oceanogr., 31, 848-858.

Burns C.W. 1969. - Relation between filtering rate, temperature and body size in four species of Daphnia. Limnol. and Oceanogr. $14: 693-700$.

Burns C.W. 1968. - The relationship between body size of filter feeding Cladocera and the maximum size of particle ingested. Limnol and Oceanogr. $13: 675-678$.

Chérifi O. 1990. - Etude du phytoplancton et de la physicochimie du lac de barrage Lalla Takerkoust. Mémoire Univ. Cadi Ayyad, Marrakech, 42 p.

Chérifi O. 1992. - Evolution et dynamique du phytoplancton en relation avec certains paramètres biotiques au niveau de la retenue Lalla Takerkoust (Maroc). Thèse $3^{e}$ cycle. Fac. Sc. Semlalia. Marrakech : $155 \mathrm{p}$.

Cheson J. 1978. - Measuring preference in selective predation. Ecology, $59: 211-215$.

De Mott R.W. 1982. - Feeding selectivities and relative ingestion rates of Daphnia and Bosmina. Limnol. and Oceanogr., 17 (3) : 518-527.

Edmondson W.T. 1957. - Trophic relations of the zooplankton. Trans. Amer. Micr. Soc., p : 225-245.

Fretwell S.D. 1987. - Food chain dynamics. The central theory of Ecology. Oikos, $50: 291-303$.

Gawler M., Blanc P., Druart J.C. \& Pelletier J.P. 1986. — Dynamiques de quelques populations majeures du phytoplancton printanier du lac Léman en relation avec le broutage et les sels nutritif. Coll. C.N.R.S. " Biologie des populations", Lyon, 4-6 septembre. Univ. Claude Bernard, Lyon, France : 412-419.

Gliwicz Z.M. \& Siedlar E. 1980. - Food size limitation and algae interfering with food collection in Daphnia. Arch. Hydrobiol., 88 (2) : 155-177.

Gliwicz Z.M. 1967. - The contribution of nannoplankton in pelagial primary production in some lakes with varying trophy. Bull. Acad. Pol. Sci., $15: 343-347$.

Gliwicz Z.M. 1969. - Studies on the feeding of pelagic zooplankton in lakes with varying trophy. Ekol. Pol., A 17 : 633-708.

Gliwicz Z.M. 1977. - Food size selection and seasonal succession of filter feeding zooplankton in a eutrophic lake. Ekol. Pol., $25: 179-225$.

Guissani G. 1974. - Predazione selettiva del coregone bondella (Coregonus sp.) de Lago Maggiore. Mem. Ist. Ital. Idrobiol., $31: 181-203$.
Hessen O.D., Andersen T. \& Lynche A. 1989. - Differential grazing and resource utilisation of zooplankton in a humic lake. Arch. Hydrobiol. 114 : 321-347.

Hutchinson G.E. 1971. - The effect of fish predation on the zooplankton of tea Adirondack Lakes, with particular reference to the alewife, Alosa pseudoharenqus. Trans. Amer. fish. Soc., 100, 2 : 325-335.

Hynes H.B.N. 1950. - The food of fresh water stiklebacks (Gasterosteus aculeatus and Pigosteus pungitus) with a review of methods used in studies of the food of fishes. J. Anim. Ecol., $19,1: 36-58$.

Ivlev V.S. 1961. - Experimental ecology of the feeding of fishs. Yale. Univ. Press. New Haven, Conn. : 302 p.

Jacobs J. 1974. — Evolution and Ecology of zooplankton communities. Special symposium volume 3. American Society of Limnology and Oceanography. Kerfoot C. edit. London, England, 1980,

Korinek V., Krepelova B. \& Machacek J. 1980. - Ecological significance of filtering structures in cladocera II. Species of the genera Daphnia and Ceriodaphnia. Int. Ver. Theor. Angew. Limnol. Verh. $21: 1567$.

Lampert W. 1974. - A method for determining food selection by zooplankton. Limnol. and Oceanogr., 19 : 995-998.

Lampert W. 1978. - A field study on the dependence of the fecundity of Daphnia sp. on food concentration. Oecologia $36: 363-369$.

Lazarro X. 1987. - A review of planktivorous fishes : their evolution, feeding behaviour, selectivities and impacts. Hydrobiologia, 146 : 97-168.

Lechowicz M.J. 1982. - The sampling characteristics of selectivity indices. Oecologia, $52: 22-30$.

Morgan N.C. et al. 1980. - Secondary production in the functionning of freshwater ecosystems. IBP hand book 22, edit. Lecren E.D. \& Lowe M.C. \& Connell R.H., Camb. Univ. P1.

Obrien W.J. \& Vinyard G.L. 1978. - Polymorphism and predation: The effect of invertebrate predation on the distribution of two Daphnia carinata varieties in south India ponds. Limnol. Oceanogr., 23 : 452-460.

Paloheimo J.E. 1979. - Indices of food preference by a predator. J. Fish. Res. Bd. Can., $36: 470-473$.

Pejler B. 1983. - Zooplanktonic indicators of trophy and their food. Hydrobiologia, $101: 111-114$.

Pikryl I. 1980. - Determination of zooplankton biomass on the basis of the lenght. Weight relations. Bull. Vurh. coduany : 13-18.

Pont D. 1983. - Recherches qualitatives sur le peuplement des Copépodes, Cladocères et Ostracodes des rizières de Camargue. Thèse Doct. Sc. Nat., Univ. de Provence, Marseille : $353 \mathrm{p}$.

Pourriot R. \& Champs P. 1982. - Consommateurs et production secondaire, 2 : 49-112. In Pourriot et al. : Ecologie du plancton des eaux continentales, Masson éd., Paris.

Pourriot R., Capblancq J., Champs P. \& Meyers J.A. 1982. Ecologie du plancton des equx continentales. Masson éd. Paris, $198 \mathrm{p}$.

Reynolds C.S. 1984. - The ecology of freshwater phytoplankton, Cambridge Univ. Pr., 384 p.

Sommer U. 1985. - Comparison between steady state and nonsteady state competition : experiments with natural phytoplankton. Limnol. Oceanogr. $30: 335-346$. 
Sommer U., Gliwicz Z.M., Lampert W. \& Duncan A. 1986. The PEG-model of seasonal succession of planktonic events in fresh waters. Arch. Hydrobiol. $106: 433-471$.

Spurles W.G. \& Holtby L.B. 1979. — Body size and feeding ecology as alternatives to taxonomy for the study of limnetic zooplankton community structure. J. fish. Res, Board Can. 36 : 1354-1363.
Strauss R.E. 1979. - Reliability estimates for Ivlev's electivity Index, the forage Ratio and a proposed linear Index of food selection. Trans. Amer. fish. Soc., $108: 344352$.

Tifnouti A., Pourriot R. \& Rougier C. 1993. - Influence de la température sur le développement et la fécondité de quatre espèces de Cladocères planctoniques (Crustacés) en présence de ressources naturelles. Annls Limnol. 29 (1) : 3-13. 